\title{
Some Integral Inequalities of Simpson Type for Strongly Extended $s$-Convex Functions
}

\author{
Yixuan Sun, Hongping Yin \\ College of Mathematics, Inner Mongolia University for Nationalities, Tongliao, China \\ Email: sunyixuan6688@qq.com, hongpingyin@qq.com
}

How to cite this paper: Sun, Y.X. and Yin, H.P. (2016) Some Integral Inequalities of Simpson Type for Strongly Extended $s$-Convex Functions. Advances in Pure Mathematics, 6, 745-753.

http://dx.doi.org/10.4236/apm.2016.611060

Received: September 20, 2016

Accepted: October 5, 2016

Published: October 8, 2016

Copyright $\odot 2016$ by authors and Scientific Research Publishing Inc. This work is licensed under the Creative Commons Attribution International License (CC BY 4.0).

http://creativecommons.org/licenses/by/4.0/

\begin{abstract}
The main purpose of this survey paper is to point out some very recent developments on Simpson's inequality for strongly extended $s$-convex function. Firstly, the concept of strongly extended $s$-convex function is introduced. Next a new identity is also established. Finally, by this identity and Hölder's inequality, some new Simpson type for the product of strongly extended $s$-convex function are obtained.
\end{abstract}

\section{Keywords}

Simpson Type Inequality, Integral Identity, Strongly Extended $s$-Convex Function

\section{Introduction}

Convex function is a kind of important function and has wide applications in pure and applied mathematics [1]. Since convex analysis appeared in 1960s, there has been tremendous interest in generalizing convex function [2]. In recent years, the generalized convex function and its application have been hot issues. The main purpose of this survey paper is to point out some very recent developments on Simpson's inequality for strongly extended s-convex function.

First, some definitions concerning various convex functions are listed.

Definition 1.1. A function $f: I \subseteq R=(-\infty, \infty) \rightarrow R$ is said to be convex if

$$
f(\lambda x+(1-\lambda) y) \leq \lambda f(x)+(1-\lambda) f(y)
$$

holds for all $x, y \in I$ and $\lambda \in[0,1]$.

The $s$-convex function was defined in [3] as follows.

Definition 1.2. A function $f: I \subseteq R \rightarrow R_{0}=[0, \infty)$ is said to be $s$-convex if

$$
f(\lambda x+(1-\lambda) y) \leq \lambda^{s} f(x)+(1-\lambda)^{s} f(y)
$$


for some $s \in(0,1]$, where $x, y \in I, \lambda \in[0,1]$.

If $s=1$, the $s$-convex function becomes a convex function on $R_{0}$.

In [4], the authors introduced the class of real functions of extended $s$-convex, defined as follows.

Definition 1.3. ([4]). A function $f: I \subseteq R \rightarrow R_{0}$ is said to be extended $s$-convex if

$$
f(\lambda x+(1-\lambda) y) \leq \lambda^{s} f(x)+(1-\lambda)^{s} f(y)
$$

for some $s \in[-1,1]$, where $x, y \in I, \lambda \in(0,1)$.

In [5] the concept of strongly convex functions below was innovated.

Definition 1.4. ([5]) A function $f:[a, b] \rightarrow R$ is said to be strongly convex with modulus $c>0$, if

$$
f(\lambda x+(1-\lambda) y) \leq \lambda f(x)+(1-\lambda) f(y)-c \lambda(1-\lambda)(x-y)^{2}
$$

is valid for all $x, y \in[a, b], \lambda \in[0,1]$.

In [6] the concept of strongly $s$-convex functions was introduced as follows.

Definition 1.5. A function $f: I \subseteq R \rightarrow R_{0}$ is said to be strongly $s$-convex with modulus $c>0$, and some $s \in(0,1]$ if

$$
f(\lambda x+(1-\lambda) y) \leq \lambda^{s} f(x)+(1-\lambda)^{s} f(y)-c \lambda(1-\lambda)(x-y)^{2}
$$

is valid all $x, y \in[a, b], \lambda \in[0,1]$.

The following inequalities of Hermite-Hadamard type were established for some of the above convex functions.

Theorem 1.1. ([7]). Let $f: I^{\circ} \subseteq R \rightarrow R$ be differentiable on $I^{\circ}, a, b \in I^{\circ}$ with $a<b$.

(1) If $\left|f^{\prime}\right|$ is convex function on $[a, b]$, then

$$
\left|\frac{f(a)+f(b)}{2}-\frac{1}{b-a} \int_{a}^{b} f(x) \mathrm{d} x\right| \leq \frac{(b-a)\left(\left|f^{\prime}(a)\right|+\left|f^{\prime}(b)\right|\right)}{8} .
$$

(2) If $\left|f^{\prime \mid}\right|^{p /(p-1)}$ is convex function on $[a, b], \quad p>1$, then

$$
\begin{aligned}
& \left|\frac{f(a)+f(b)}{2}-\frac{1}{b-a} \int_{a}^{b} f(x) \mathrm{d} x\right| \\
& \leq \frac{b-a}{2(p+1)^{1 / p}}\left(\frac{\left|f^{\prime}(a)\right|^{p /(p-1)}+\left|f^{\prime}(b)\right|^{p /(p-1)}}{2}\right)^{(p-1) / p} .
\end{aligned}
$$

Theorem 1.2. ([8]). Let $f: I \subset R \rightarrow R$ be differentiable on $I^{\circ}, a, b \in I^{\circ}$ with $a<b$. If $\left|f^{\prime}\right|^{q}$ is $s$-convex function on $[a, b]$ for some fixed $s \in(0,1]$ and $q \geq 1$, then

$$
\begin{aligned}
& \left|\frac{f(a)+f(b)}{2}-\frac{1}{b-a} \int_{a}^{b} f(x) \mathrm{d} x\right| \\
& \leq \frac{b-a}{2}\left(\frac{1}{2}\right)^{1-1 / a}\left[\frac{2+1 / 2^{s}}{(s+1)(s+2)}\right]\left(\left|f^{\prime}(a)\right|^{q}+\left|f^{\prime}(b)\right|^{q}\right)^{1 / a} .
\end{aligned}
$$


Theorem 1.3. ([9]). Let $f: I \subset R_{0} \rightarrow R$ be differentiable on $I^{\circ}, a, b \in I^{\circ}$ with $a<b$, and $f^{\prime} \in L[a, b]$. If $\left|f^{\prime}\right|$ is $s$-convex function on $[a, b]$ for some fixed $s \in(0,1]$, then

$$
\begin{aligned}
& \left|\frac{1}{6}\left[f(a)+f(b)+4 f\left(\frac{a+b}{2}\right)\right]-\frac{1}{b-a} \int_{a}^{b} f(x) \mathrm{d} x\right| \\
& \leq \frac{(s-4) 6^{s+1}+2 \times 5^{s+2}-2 \times 3^{s+2}+2}{6^{s+2}(s+1)(s+2)}(b-a)\left(\left|f^{\prime}(a)\right|+\left|f^{\prime}(b)\right|\right) .
\end{aligned}
$$

In [6], Ju Hua et al. established the following theorem.

Theorem 1.4. Let $f: I \subseteq R_{0} \rightarrow R_{0}$ be differentiable mapping on $I^{\circ}$ and $a, b \in I$ with $a<b$. If $f^{\prime \prime} \in(L[a, b])$ and $\left|f^{\prime \prime}\right|^{q}$ is strongly s-convex on $[a, b]$ for $q \geq 1$, $s \in(0,1]$, then

$$
\begin{aligned}
& \left|\frac{1}{6}\left[f(a)+2 f\left(\frac{2 a+b}{3}\right)+2 f\left(\frac{a+2 b}{3}\right)+f(b)\right]-\frac{1}{b-a} \int_{a}^{b} f(x) \mathrm{d} x\right| \\
& \leq \frac{6^{1 / a}(b-a)^{2}}{324}\left\{\left[\frac{(s-3) 3^{s+2}+(s+7) 2^{s+2}}{3^{s}(s+1)(s+2)(s+3)}\left|f^{\prime \prime}(a)\right|^{q}\right.\right. \\
& \left.\quad+\frac{1}{3^{s}(s+2)(s+3)}\left|f^{\prime \prime}(b)\right|^{q}-\frac{c(b-a)^{2}}{45}\right]^{1 / a} \\
& +\left[\frac{(s-1) 2^{s+2}+s+5}{3^{s}(s+1)(s+2)(s+3)}\left(\left|f^{\prime \prime}(a)\right|^{q}+\left|f^{\prime \prime}(b)\right|^{q}\right)-\frac{11 c(b-a)^{2}}{270}\right]^{1 / q} \\
& +\left[\frac{1}{3^{s}(s+2)(s+3)}\left|f^{\prime \prime}(a)\right|^{q}\right. \\
& \left.\left.+\frac{(s-3) 3^{s+2}+(s+7) 2^{s+2}}{3^{s}(s+1)(s+2)(s+3)}\left|f^{\prime \prime}(b)\right|^{q}-\frac{c(b-a)^{2}}{45}\right]^{1 / q}\right\} .
\end{aligned}
$$

In this paper, the authors introduce the concept of strongly extended $s$-convex function and establish a new identity. By this identity and Hölder's inequality, some new Simpson type for the product of strongly extended $s$-convex function and discussed and some results are obtained.

\section{Definition and Integral Identities}

Now the concept of strongly extended $s$-convex function is introduced.

Definition 2.1. A function $f:[a, b] \rightarrow R$ is said to be strongly extended s-convex with modulus $c>0$, if

$$
f(t x+(1-t) y) \leq t^{s} f(x)+(1-t)^{s} f(y)-c t(1-t)(x-y)^{2}
$$

is valid for all $x, y \in[a, b]$ and $t \in(0,1)$, some $s \in[-1,1]$.

For establishing new integral inequalities of Simpson type involving the strongly extended s-convex function, the following identity is needed:

Lemma 2.1. Let $f: I \subseteq R \rightarrow R$ be differentiable on $I^{\circ}$ and where $a, b \in I$ with 
$a<b$. If $f^{\prime} \in L([a, b])$, then the following identity holds:

$$
\begin{aligned}
& \frac{1}{4}\left[f(a)+f\left(\frac{2 a+b}{3}\right)+f\left(\frac{a+2 b}{3}\right)+f(b)\right]-\frac{1}{b-a} \int_{a}^{b} f(x) \mathrm{d} x \\
& =\frac{b-a}{9}\left[\int_{0}^{1}\left(t-\frac{3}{4}\right) f^{\prime}\left((1-t) a+t \frac{2 a+b}{3}\right) \mathrm{d} t\right. \\
& \quad+\int_{0}^{1}\left(t-\frac{2}{4}\right) f^{\prime}\left((1-t) \frac{2 a+b}{3}+t \frac{a+2 b}{3}\right) \mathrm{d} t \\
& \left.\quad+\int_{0}^{1}\left(t-\frac{1}{4}\right) f^{\prime}\left((1-t) \frac{a+2 b}{3}+t b\right) \mathrm{d} t\right] .
\end{aligned}
$$

Proof. By straightforward computation, the result is followed. The proof is completed.

Lemma 2.2. ([4]). Let $f: I \subseteq R \rightarrow R$ be differentiable on $I^{\circ}$ and $a, b \in I$ with $a<b$. If $f^{\prime} \in L([a, b])$, then

$$
\begin{aligned}
& f\left(\frac{a+b}{2}\right)-\frac{1}{b-a} \int_{a}^{b} f(x) \mathrm{d} x \\
& =(b-a)\left(\int_{0}^{\frac{1}{2}} t f^{\prime}(t a+(1-t) b) \mathrm{d} t+\int_{\frac{1}{2}}^{1}(1-t) f^{\prime}(t a+(1-t) b) \mathrm{d} t\right) .
\end{aligned}
$$

\section{Some Integral Inequalities of Simpson Type}

Theorem 3.1. Let $f: I \subseteq R \rightarrow R_{0}$ be differentiable mapping on $I^{\circ}$ and $a, b \in I$ with $a<b$. If $f^{\prime} \in L([a, b])$ and $\left|f^{\prime}\right|^{q}$ is strongly extended s-convex on $[a, b]$ for $q \geq 1$, $s \in(-1,1]$, then

$$
\begin{aligned}
& \left|\frac{1}{4}\left[f(a)+f\left(\frac{2 a+b}{3}\right)+f\left(\frac{a+2 b}{3}\right)+f(b)\right]-\frac{1}{b-a} \int_{a}^{b} f(x) \mathrm{d} x\right| \\
& \leq \frac{b-a}{9 \times 16^{1-1 / q}}\left\{5 ^ { 1 - 1 / a } \left[\frac{3 \times 2^{2 s+1} s+2^{2 s+2}+1}{2^{2 s+3}(s+1)(s+2)}\left|f^{\prime}(a)\right|^{q}\right.\right. \\
& \left.+\frac{3^{s+2}-2^{2 s+2}+2^{2 s+1} s}{2^{2 s+3}(s+1)(s+2)}\left|f^{\prime}\left(\frac{2 a+b}{3}\right)\right|^{q}-\frac{71 c(b-a)^{2}}{13824}\right]^{1 / a} \\
& +4^{1-1 / q}\left[\frac{2^{s+1}+1}{2^{s+1}(s+1)(s+2)}\left(\left|f^{\prime}\left(\frac{2 a+b}{3}\right)\right|^{q}+\left|f^{\prime}\left(\frac{a+2 b}{3}\right)\right|^{q}\right)-\frac{c(b-a)^{2}}{288}\right]^{1 / q} \\
& +5^{1-1 / q}\left[\frac{3^{s+2}-2^{2 s+2}+2^{2 s+1} s}{2^{2 s+3}(s+1)(s+2)}\left|f^{\prime}\left(\frac{a+2 b}{3}\right)\right|^{q}\right. \\
& \left.\left.+\frac{3 \times 2^{2 s+1} s+2^{2 s+2}+1}{2^{2 s+3}(s+1)(s+2)}\left|f^{\prime}(b)\right|^{q}-\frac{71 c(b-a)^{2}}{13824}\right]^{1 / q}\right\} .
\end{aligned}
$$

Proof. Using Lemma 2.1 and by Hölder's inequality, the followings can be obtained: 


$$
\begin{aligned}
& \left|\frac{1}{4}\left[f(a)+f\left(\frac{2 a+b}{3}\right)+f\left(\frac{a+2 b}{3}\right)+f(b)\right]-\frac{1}{b-a} \int_{a}^{b} f(x) \mathrm{d} x\right| \\
& \leq \frac{b-a}{9}\left[\int_{0}^{1}\left|t-\frac{3}{4}\right|\left|f^{\prime}\left((1-t) a+t \frac{2 a+b}{3}\right)\right| \mathrm{d} t\right. \\
& \left.+\int_{0}^{1}\left|t-\frac{2}{4}\right|\left|f^{\prime}\left((1-t) \frac{2 a+b}{3}+t \frac{a+2 b}{3}\right)\right| \mathrm{d} t+\int_{0}^{1}\left|t-\frac{1}{4}\right|\left|f^{\prime}\left((1-t) \frac{a+2 b}{3}+t b\right)\right| \mathrm{d} t\right] \\
& \leq \frac{b-a}{9}\left[\left(\int_{0}^{1}\left|t-\frac{3}{4}\right| \mathrm{d} t\right)^{1-1 / a}\left(\left.\int_{0}^{1}\left|t-\frac{3}{4}\right|\left|f^{\prime}\left((1-t) a+t \frac{2 a+b}{3}\right)\right|\right|^{q} \mathrm{~d} t\right)^{1 / q}\right. \\
& +\left(\int_{0}^{1}\left|t-\frac{2}{4}\right| \mathrm{d} t\right)^{1-1 / a}\left(\int_{0}^{1}\left|t-\frac{2}{4}\right|\left|f^{\prime}\left((1-t) \frac{2 a+b}{3}+t \frac{a+2 b}{3}\right)\right|^{q} \mathrm{~d} t\right)^{1 / a} \\
& \left.+\left(\int_{0}^{1}\left|t-\frac{1}{4}\right| \mathrm{d} t\right)^{1-1 / a}\left(\int_{0}^{1}\left|t-\frac{1}{4}\right|\left|f^{\prime}\left((1-t) \frac{a+2 b}{3}+t b\right)\right| \mathrm{d} t\right)^{q}\right],
\end{aligned}
$$

where,

$$
\begin{aligned}
& \int_{0}^{1}\left|t-\frac{3}{4}\right| \mathrm{d} t=\frac{5}{16}, \int_{0}^{1} t(1-t)\left|t-\frac{3}{4}\right| \mathrm{d} t=\frac{71}{1536}, \int_{0}^{1}\left|t-\frac{2}{4}\right| \mathrm{d} t=\frac{1}{4} \\
& \int_{0}^{1} t(1-t)\left|t-\frac{2}{4}\right| \mathrm{d} t=\frac{1}{32}, \int_{0}^{1}\left|t-\frac{1}{4}\right| \mathrm{d} t=\frac{5}{16}, \int_{0}^{1} t(1-t)\left|t-\frac{1}{4}\right| \mathrm{d} t=\frac{71}{1536} .
\end{aligned}
$$

Again $\left|f^{\prime}\right|^{q}$ is strongly extended $s$-convex on $[a, b]$, so

$$
\begin{aligned}
& \left.\int_{0}^{1}\left|t-\frac{3}{4}\right| f^{\prime}\left((1-t) a+t \frac{2 a+b}{3}\right)\right|^{q} \mathrm{~d} t \\
& \leq \int_{0}^{1}\left|t-\frac{3}{4}\right|\left((1-t)^{s}\left|f^{\prime}(a)\right|^{q}+t^{s}\left|f^{\prime}\left(\frac{2 a+b}{3}\right)\right| \mathrm{d} t\right. \\
& \quad-\frac{c(b-a)^{2}}{9} \int_{0}^{1} t(1-t)\left|t-\frac{3}{4}\right| \mathrm{d} t \\
& =\frac{3 \times 2^{2 s+1} s+2^{2 s+2}+1}{2^{2 s+3}(s+1)(s+2)}\left|f^{\prime}(a)\right|^{q} \\
& \quad+\frac{3^{s+2}-2^{2 s+2}+2^{2 s+1} s}{2^{2 s+3}(s+1)(s+2)}\left|f^{\prime}\left(\frac{2 a+b}{3}\right)\right|^{q}-\frac{71 c(b-a)^{2}}{13824} . \\
& \int_{0}^{1}\left|t-\frac{2}{4}\right|\left|f^{\prime}\left((1-t) \frac{2 a+b}{3}+t \frac{a+2 b}{3}\right)\right|^{q} \mathrm{~d} t \\
& \leq \int_{0}^{1}\left|t-\frac{2}{4}\right|\left((1-t)^{s}\left|f^{\prime}\left(\frac{2 a+b}{3}\right)\right|^{q}+t^{s}\left|f^{\prime}\left(\frac{a+2 b}{3}\right)\right|^{q}\right) \mathrm{d} t \\
& \quad-\frac{c(b-a)^{2}}{9} \int_{0}^{1} t(1-t)\left|t-\frac{2}{4}\right| \mathrm{d} t \\
& =\frac{2^{s+1}+1}{2^{s+1}(s+1)(s+2)}\left(\left|f^{\prime}\left(\frac{2 a+b}{3}\right)\right|^{q}+\left|f^{\prime}\left(\frac{a+2 b}{3}\right)\right|^{q}\right)-\frac{c(b-a)^{2}}{288} .
\end{aligned}
$$




$$
\begin{aligned}
& \int_{0}^{1}\left|t-\frac{1}{4}\right|\left|f^{\prime}\left((1-t) \frac{a+2 b}{3}+t b\right)\right|^{q} \mathrm{~d} t \\
& \leq \int_{0}^{1}\left|t-\frac{1}{4}\right|\left((1-t)^{s}\left|f^{\prime}\left(\frac{a+2 b}{3}\right)\right|^{q}+t^{s}\left|f^{\prime \prime}(b)\right|^{q}\right) \mathrm{d} t \\
& -\frac{c(b-a)^{2}}{9} \int_{0}^{1} t(1-t)\left|t-\frac{1}{4}\right| \mathrm{d} t \\
& =\frac{3^{s+2}-2^{2 s+2}+2^{2 s+1} s}{2^{2 s+3}(s+1)(s+2)}\left|f^{\prime}\left(\frac{a+2 b}{3}\right)\right|^{q} \\
& +\frac{3 \times 2^{2 s+1} s+2^{2 s+2}+1}{2^{2 s+3}(s+1)(s+2)}\left|f^{\prime}(b)\right|^{q}-\frac{71 c(b-a)^{2}}{13824} .
\end{aligned}
$$

Substituting the above (3.3)-(3.6) into the inequality (3.2) results in the inequality (3.1).

Theorem 3.1 is proved.

Corollary 3.2. Under conditions of Theorem 3.1, if $q=1$, then

$$
\begin{aligned}
& \left|\frac{1}{4}\left[f(a)+f\left(\frac{2 a+b}{3}\right)+f\left(\frac{a+2 b}{3}\right)+f(b)\right]-\frac{1}{b-a} \int_{a}^{b} f(x) \mathrm{d} x\right| \\
& \leq \frac{b-a}{9}\left\{\frac{3 \times 2^{2 s+1} s+2^{2 s+2}+1}{2^{2 s+3}(s+1)(s+2)}\left|f^{\prime}(a)\right|\right. \\
& \quad+\frac{3^{s+2}+2^{2 s+3}-2^{2 s+2}+2^{s+2}+2^{2 s+1} s}{2^{2 s+3}(s+1)(s+2)}\left|f^{\prime}\left(\frac{2 a+b}{3}\right)\right| \\
& \quad+\frac{3^{s+2}+2^{2 s+3}-2^{2 s+2}+2^{s+2}+2^{2 s+1} s}{2^{2 s+3}(s+1)(s+2)}\left|f^{\prime}\left(\frac{a+2 b}{3}\right)\right| \\
& \left.\quad+\frac{3 \times 2^{2 s+1} s+2^{2 s+2}+1}{2^{2 s+3}(s+1)(s+2)}\left|f^{\prime}(b)\right|-\frac{95 c(b-a)^{2}}{6912}\right\} .
\end{aligned}
$$

Theorem 3.3. Let $f: I \subseteq R \rightarrow R_{0}$ be differentiable mapping on $I^{\circ}$ and $a, b \in I$ with $a<b$. If $f^{\prime} \in L([a, b])$ and $\left|f^{\prime}\right|$ is strongly extended s-convex on $[a, b]$ for $q \geq 1, \quad s=-1$, then

$$
\begin{aligned}
& \left|f\left(\frac{a+b}{2}\right)-\frac{1}{b-a} \int_{a}^{b} f(x) \mathrm{d} x\right| \\
& \leq \frac{b-a}{8^{1-1 / q}}\left\{\left[\frac{\left|f^{\prime}(a)\right|^{q}+(2 \ln 2-1)\left|f^{\prime}(b)\right|^{q}}{2}-\frac{5 c(b-a)^{2}}{192}\right]^{1 / q}\right. \\
& \left.\quad+\left[\frac{(2 \ln 2-1)\left|f^{\prime}(a)\right|^{q}+\left|f^{\prime}(b)\right|^{q}}{2}-\frac{5 c(b-a)^{2}}{192}\right]^{1 / q}\right\} .
\end{aligned}
$$

Proof. Since $\left|f^{\prime}\right|^{q}$ is strongly extended $s$-convex on $[a, b]$, using Lemma 2.2 and by Hölder's inequality, the followings can be obtained: 


$$
\begin{aligned}
& \left|f\left(\frac{a+b}{2}\right)-\frac{1}{b-a} \int_{a}^{b} f(x) \mathrm{d} x\right| \\
& \leq(b-a)\left[\int_{0}^{1 / 2} t\left|f^{\prime}(t a+(1-t) b)\right| \mathrm{d} t+\int_{1 / 2}^{1}(1-t)\left|f^{\prime}(t a+(1-t) b)\right| \mathrm{d} t\right] \\
& \leq(b-a)\left[\left(\int_{0}^{1 / 2} t \mathrm{~d} t\right)^{1-1 / q}\left(\int_{0}^{1 / 2} t\left|f^{\prime}(t a+(1-t) b)\right|^{q} \mathrm{~d} t\right)^{1 / q}\right. \\
& +\left(\int_{1 / 2}^{1}(1-t) \mathrm{d} t\right)^{1-1 / q}\left(\int_{1 / 2}^{1}(1-t)\left|f^{\prime}(t a+(1-t) b)\right|^{q} \mathrm{~d} t\right)^{1 / q} \\
& \leq \frac{b-a}{8^{1-1 / q}}\left\{\left[\int_{0}^{1 / 2} t\left(t^{-1}\left|f^{\prime}(a)\right|^{q}+(1-t)^{-1}\left|f^{\prime}(b)\right|^{q}-c t(1-t)(b-a)^{2}\right) \mathrm{d} t\right]^{1 / q}\right. \\
& \left.+\left[\int_{1 / 2}^{1}(1-t)\left(t^{-1}\left|f^{\prime}(a)\right|^{q}+(1-t)^{-1}\left|f^{\prime}(b)\right|^{q}-c t(1-t)(b-a)^{2}\right) \mathrm{d} t\right]^{1 / q}\right\} \\
& =\frac{b-a}{8^{1-1 / q}}\left\{\left[\frac{\left|f^{\prime}(a)\right|^{q}+(2 \ln 2-1)\left|f^{\prime}(b)\right|^{q}}{2}-\frac{5 c(b-a)^{2}}{192}\right]^{1 / q}\right. \\
& \left.+\left[\frac{(2 \ln 2-1)\left|f^{\prime}(a)\right|^{q}+\left|f^{\prime}(b)\right|^{q}}{2}-\frac{5 c(b-a)^{2}}{192}\right]^{1 / q}\right\} .
\end{aligned}
$$

Theorem 3.3 is proved.

Theorem 3.4. Let $f: I \subseteq R \rightarrow R_{0}$ be differentiable mapping on $I^{\circ}$ and $a, b \in I$ with $a<b$. If $f^{\prime} \in L([a, b])$ and $\left|f^{\prime}\right|^{q}$ is strongly extended s-convex on $[a, b]$ for $q>1, \quad s \in(-1,1]$, then

$$
\begin{aligned}
& \left|\frac{1}{4}\left[f(a)+f\left(\frac{2 a+b}{3}\right)+f\left(\frac{a+2 b}{3}\right)+f(b)\right]-\frac{1}{b-a} \int_{a}^{b} f(x) \mathrm{d} x\right| \\
& \leq \frac{b-a}{9}\left(\frac{q-1}{2 q-1}\right)^{1-1 / q}\left[\left(\frac{1+3^{(2 q-1) /(q-1)}}{4^{(2 q-1) /(q-1)}}\right)^{1-1 / q}\right. \\
& \quad \times\left(\frac{1}{s+1}\left|f^{\prime}(a)\right|^{q}+\frac{1}{s+1}\left|f^{\prime}\left(\frac{2 a+b}{3}\right)\right|^{q}-\frac{c(b-a)^{2}}{54}\right)^{1 / q} \\
& +\frac{1}{2}\left(\frac{1}{s+1}\left|f^{\prime}\left(\frac{2 a+b}{3}\right)\right|^{q}+\frac{1}{s+1}\left|f^{\prime}\left(\frac{a+2 b}{3}\right)\right|^{q}-\frac{c(b-a)^{2}}{54}\right)^{1 / q} \\
& \left.+\left(\frac{1+3^{(2 q-1) /(q-1)}}{4^{(2 q-1) /(q-1)}}\right)^{1-1 / q}\left[\frac{1}{s+1}\left|f^{\prime}\left(\frac{a+2 b}{3}\right)\right|^{q}+\frac{1}{s+1}\left|f^{\prime}(b)\right|^{q}-\frac{c(b-a)^{2}}{54}\right]^{1 / q}\right\} .
\end{aligned}
$$

Proof. By the Lemma 2.1 and using Hölder's inequality, the followings can be obtained: 


$$
\begin{aligned}
& \left|\frac{1}{4}\left[f(a)+f\left(\frac{2 a+b}{3}\right)+f\left(\frac{a+2 b}{3}\right)+f(b)\right]-\frac{1}{b-a} \int_{a}^{b} f(x) \mathrm{d} x\right| \\
& \leq \frac{b-a}{9}\left[\left(\int_{0}^{1}\left|t-\frac{3}{4}\right|^{q /(q-1)} \mathrm{d} t\right)^{1-1 / a}\left(\int_{0}^{1}\left|f^{\prime}\left((1-t) a+t \frac{2 a+b}{3}\right)\right|^{q} \mathrm{~d} t\right)^{1 / a}\right. \\
& \quad+\left(\int_{0}^{1}\left|t-\frac{2}{4}\right|^{q /(q-1)} \mathrm{d} t\right)^{1-1 / q}\left(\int_{0}^{1}\left|f^{\prime}\left((1-t) \frac{2 a+b}{3}+t \frac{a+2 b}{3}\right)\right|^{q} \mathrm{~d} t\right)^{1 / q} \\
& \left.\quad+\left(\int_{0}^{1}\left|t-\frac{1}{4}\right|^{q /(q-1)} \mathrm{d} t\right)^{1-1 / a}\left(\int_{0}^{1}\left|f^{\prime}\left((1-t) \frac{a+2 b}{3}+t b\right)\right|^{q} \mathrm{~d} t\right)^{1 / q}\right],
\end{aligned}
$$

where,

$$
\begin{aligned}
& \int_{0}^{1}\left|t-\frac{3}{4}\right|^{q /(q-1)} \mathrm{d} t=\int_{0}^{1}\left|t-\frac{1}{4}\right|^{q /(q-1)} \mathrm{d} t=\frac{\left(1+3^{(2 q-1) /(q-1)}\right)(q-1)}{4^{(2 q-1) /(q-1)}(2 q-1)}, \\
& \int_{0}^{1}\left|t-\frac{2}{4}\right|^{q /(q-1)} \mathrm{d} t=\frac{q-1}{2^{q /(q-1)}(2 q-1)} .
\end{aligned}
$$

Since $\left|f^{\prime}\right|^{q}$ is strongly extended $s$-convex on $[a, b]$, so

$$
\begin{aligned}
& \int_{0}^{1}\left|f^{\prime}\left((1-t) a+t \frac{2 a+b}{3}\right)\right|^{q} \mathrm{~d} t \\
& \leq \frac{1}{s+1}\left|f^{\prime}(a)\right|^{q}+\frac{1}{s+1}\left|f^{\prime}\left(\frac{2 a+b}{3}\right)\right|^{q}-\frac{c(b-a)^{2}}{54}, \\
& \left.\int_{0}^{1}\left|f^{\prime}\left((1-t) \frac{2 a+b}{3}+t \frac{a+2 b}{3}\right)\right|\right|^{q} \mathrm{~d} t \\
& \leq \frac{1}{s+1}\left|f^{\prime}\left(\frac{2 a+b}{3}\right)\right|^{q}+\frac{1}{s+1}\left|f^{\prime}\left(\frac{a+2 b}{3}\right)\right|^{q}-\frac{c(b-a)^{2}}{54}, \\
& \int_{0}^{1}\left|f^{\prime}\left((1-t) \frac{a+2 b}{3}+t b\right)\right| \mathrm{d} t \\
& \leq \frac{1}{s+1}\left|f^{\prime}\left(\frac{a+2 b}{3}\right)\right|^{q}+\frac{1}{s+1}\left|f^{\prime}(b)\right|^{q}-\frac{c(b-a)^{2}}{54} .
\end{aligned}
$$

Substituting (3.10)-(3.13) into the inequality (3.9) yields (3.8). Theorem 3.4 is proved.

\section{Conclusion}

In this paper, the authors introduce the concept of strongly extended s-convex function and establish a new identity. Then by this identity and Hölder's inequality, some new Simpson type for the product of strongly extended s-convex function are obtained.

\section{Acknowledgements}

This work was supported by the National Natural Science Foundation of China No. 11361038 and by the Inner Mongolia Autonomous Region Natural Science Foundation 
Project under Grant No. 2015MS0123, China.

\section{References}

[1] Niculescu, C.P. and Persson, L.E. (2005) Convex Functions and Their Applications. CMS Books in Mathematics, Springer-Verlag.

[2] Dragomir, S.S. and Pearce, C.E.M. (2000) Selected Topics on Hermitr-Hadamard Type Inequalities and Applications, RGMIA Monographs, Victoria University. http://rgmia.org/monographs/hermite_hadamard.html

[3] Hudzik, H. and Maligranda, L. (1994) Some Remarks on s-Convex Functions. Aequationes Mathematicae, 48, 100-111. http://dx.doi.org/10.1007/BF01837981

[4] Xi, B.Y. and Qi, F. (2015) Inequalities of Hermite-Hadamard Type for Extended s-Convex Functions and Applications to Means. Journal of Nonlinear and Convex Analysis, 16, 873-890.

[5] Polyak, B.T. (1966) Existence Theorems and Convergence of Minimizing Sequences in Extremum Problems with Restrictions. Soviet Math.Dokl., 7, 72-75.

[6] Hua, J., Xi, B.Y. and Qi, F. (2015) Some New Inequalities of Simpson Type for Strongly s-Convex Functions. Afrika Matematika, 26, 741-752.

http://dx.doi.org/10.1007/s13370-014-0242-2

[7] Dragomir, S.S. and Agarwal, R.P. (1998) Two Inequalities for Differentiable Mappings and Applications to Special Means of Real Numbers and to Trapezoidal Formula. Applied Mathematics Letters, 11, 91-95. http://dx.doi.org/10.1016/S0893-9659(98)00086-X

[8] Kirmaci, U.S. (2004) Inequalities for Differentiable Mappings and Applications to Special Means of Real Numbers to Midpoint Formula. Applied Mathematics and Computation, 147, 137-146. http://dx.doi.org/10.1016/S0096-3003(02)00657-4

[9] Sarikaya, M.Z., Set, E. and Özdemir, M.E. (2010) On New Inequalities of Simpson's Type for s-Convex Functions. Computers \& Mathematics with Applications, 60, 2191-2199. http://dx.doi.org/10.1016/j.camwa.2010.07.033.

Submit or recommend next manuscript to SCIRP and we will provide best service for you:

Accepting pre-submission inquiries through Email, Facebook, LinkedIn, Twitter, etc. A wide selection of journals (inclusive of 9 subjects, more than 200 journals)

Providing 24-hour high-quality service

User-friendly online submission system

Fair and swift peer-review system

Efficient typesetting and proofreading procedure

Display of the result of downloads and visits, as well as the number of cited articles

Maximum dissemination of your research work

Submit your manuscript at: http://papersubmission.scirp.org/

Or contact apm@scirp.org 